Published in Ahmed, S. E., Carvalho, F. \& Puntanen, S. (eds.)

Matrices, Statistics and Big Data : Selected Contributions from IWMS 2016.

Contributions to Statistics. Cham: Springer. ISBN 978-3-030-17519-1.

https://doi.org/10.1007/978-3-030-17519-1_5.

\title{
A notion of positive definiteness for arithmetical functions
}

\author{
January 2, 2017 \\ Mika MatTila \\ Department of Mathematics \\ Tampere University of Technology, Finland \\ mika.mattila@tut.fi \\ Pentti HaukKanen \\ School of Information Sciences \\ University of Tampere, Finland \\ pentti.haukkanen@uta.fi
}

\begin{abstract}
In the theory of Fourier transform some functions are said to be positive definite based on the positive definiteness property of a certain class of matrices associated with these functions. In the present article we consider how to define a similar positive definiteness property for arithmetical functions, whose domain is not the set of real numbers but merely the set of positive integers. After finding a suitable definition for this concept we shall use it to construct a partial ordering on the set of arithmetical functions. We shall study some of the basic properties of our newly defined relations and consider a couple of well-known arithmetical functions as examples.
\end{abstract}

Keywords: Arithmetical function, GCD matrix, Positive definite function, Positive semidefinite ordering, Dirichlet convolution, Möbius function

AMS subject classification: 11A25, 11C20, 15B36

\section{Introduction}

A complex valued function $f: \mathbb{R} \rightarrow \mathbb{C}$ is said to be a positive definite function if the matrix $\left[f\left(x_{i}-x_{j}\right)\right]$ is positive semidefinite for all choices of points $\left\{x_{1}, x_{2}, \ldots, x_{n}\right\} \subset \mathbb{R}$ and all $n=1,2, \ldots$. A positive definite function is under mild restrictions the Fourier transform of a nonnegative real valued function $g: \mathbb{R} \rightarrow \mathbb{R}_{\geq 0}$; see [3] or [5. Article 192B] for Bochner's theorem (note that the notion of a "positive semidefinite function" is not a term usually employed). By 
using the definition it is possible to prove several basic properties for a positive definite function $f$ :

- $f(-x)=\overline{f(x)}$ for all $x \in \mathbb{R}$

- $f(0) \in \mathbb{R}$ and $f(0) \geq 0$

- $f$ is a bounded function, and $|f(x)| \leq f(0) \quad \forall x \in \mathbb{R}$

- If $f$ is continuous at 0 , then it is continuous everywhere

- If $f_{1}, f_{2}, \ldots, f_{n}$ are positive definite functions and $a_{1}, a_{2}, \ldots, a_{n}$ are nonnegative real numbers, then the function $a_{1} f_{1}+a_{2} f_{2}+\cdots+a_{n} f_{n}$ is a positive definite function

- If $f$ is a positive definite function, then so are $\bar{f}$ and $|f|^{2}$

Functions $\cos x$ (but not $\sin x), e^{a i x}(a \in \mathbb{R}), \frac{1}{1-i x}, \frac{1}{1+x^{2}}$ and $\frac{1}{\cosh x}$ are all examples of positive definite functions (for more information, see [8, pp. 400-401] and [3, Section 3]).

In this article we are interested in arithmetical functions, which are realvalued (or sometimes complex-valued) functions on $\mathbb{Z}^{+}=\{1,2,3, \ldots\}$. There are various operations defined on the set of arithmetical functions, see [2, 9]. For our purposes the most important are:

- The usual sum: $(f+g)(m)=f(m)+g(m) \quad \forall m \in \mathbb{Z}^{+}$

- The usual product: $(f g)(m)=f(m) g(m) \quad \forall m \in \mathbb{Z}^{+}$

- The Dirichlet convolution: $(f * g)(m)=\sum_{d \mid m} f(d) g\left(\frac{m}{d}\right) \quad \forall m \in \mathbb{Z}^{+}$

One of the main goals of this article is to consider how to define positive definiteness property for arithmetical functions. The original definition is a bit problematic since it would require the function to be defined on negative integers as well. There are a couple of ways how one may try to get around this problem, and we shall discuss them in Section 2 In Section 3 we shall introduce our final definition and in Section 4 we investigate some of the basic properties of our newly defined positive definiteness concept. In Section 5 we use our positive definiteness relation to define a partial order on the set of arithmetical functions and then study the properties of this relation. We conclude this article by presenting examples concerning some fundamental arithmetical functions.

\section{Defining positive definiteness of arithmetical functions by using the original definition}

The most obvious way to define positive definiteness for arithmetical functions would be to expand the domain of arithmetical functions and to define the 
concept by using the matrix $\left[f\left(x_{i}-x_{j}\right)\right]$. First it should be noted that without loss of generality, we may assume that $x_{1}<x_{2}<\cdots<x_{n}$. If $x_{i}=x_{j}$ for some indices $i$ and $j$ with $i \neq j$, then the respective rows (and respective columns) are identical and the multiplicity of eigenvalue zero is increased by one. After eliminating identical rows and columns we can permute the rows and respective columns of the matrix $\left[f\left(x_{i}-x_{j}\right)\right]$ so that $x_{1}<x_{2}<\cdots<x_{n}$ is satisfied and the eigenvalues are still the same (if $P$ is any permutation matrix, then $P^{-1}=P^{T}$ and the matrices $P^{T} A P$ and $A$ share the same spectrum).

For an arithmetical function $f$ it is customary to assume that $f(x)=0$ whenever $x \notin \mathbb{Z}^{+}$. Under this assumption the matrix $\left[f\left(x_{i}-x_{j}\right)\right]$ takes the form

$$
\left[\begin{array}{cccc}
f\left(x_{1}-x_{1}\right) & f\left(x_{1}-x_{2}\right) & f\left(x_{1}-x_{3}\right) & \ldots \\
f\left(x_{2}-x_{1}\right) & f\left(x_{2}-x_{2}\right) & f\left(x_{2}-x_{3}\right) & \ldots \\
f\left(x_{3}-x_{1}\right) & f\left(x_{3}-x_{2}\right) & f\left(x_{3}-x_{3}\right) & \ldots \\
\vdots & \vdots & \vdots & \ddots
\end{array}\right]=\left[\begin{array}{cccc}
0 & 0 & 0 & \ldots \\
f\left(x_{2}-x_{1}\right) & 0 & 0 & \ldots \\
f\left(x_{3}-x_{1}\right) & f\left(x_{3}-x_{2}\right) & 0 & \ldots \\
\vdots & \vdots & \vdots & \ddots
\end{array}\right] .
$$

Since every diagonal element of this lower triangular matrix is nonnegative, the matrix is positive semidefinite. In other words, by this definition any arithmetical function $f$ is positive definite, which makes no sense.

If $f$ is a real-valued arithmetical function, then another rather obvious attempt would be to define $f(-m)=f(m)$ for all $m \in \mathbb{Z}^{+}$, which makes the matrix $\left[f\left(x_{i}-x_{j}\right)\right]$ symmetric. In this case the matrix $\left[f\left(x_{i}-x_{j}\right)\right]$ takes the form

$$
\left[\begin{array}{cccc}
f(0) & f\left(x_{2}-x_{1}\right) & f\left(x_{3}-x_{1}\right) & \cdots \\
f\left(x_{2}-x_{1}\right) & f(0) & f\left(x_{3}-x_{2}\right) & \cdots \\
f\left(x_{3}-x_{1}\right) & f\left(x_{3}-x_{2}\right) & f(0) & \cdots \\
\vdots & \vdots & \vdots & \ddots
\end{array}\right] .
$$

This is still problematic since $f(0)$ is remains undefined. However, the value $f(0)$ is crucial to the positive definiteness of the matrix $\left[f\left(x_{i}-x_{j}\right)\right]$. As was the case with the usual positive definite functions, also this definition implies that $|f(i)| \leq f(0)$ for all $i \in \mathbb{Z}^{+}$. It becomes quite clear that this approach does not work either, and therefore there seems to be no natural way to define positive definiteness of arithmetical functions by using the matrix $\left[f\left(x_{i}-x_{j}\right)\right]$. It seems that we need to use a different class of matrices in order to define positive definiteness for arithmetical functions.

\section{Defining positive definiteness by using GCD matrices}

Let $\mathcal{A}$ denote the set of arithmetical functions and let $f \in \mathcal{A}$. Let

$$
S=\left\{x_{1}, x_{2}, \ldots, x_{n}\right\}
$$

be a finite subset of $\mathbb{Z}^{+}$with $x_{1}<x_{2}<\cdots<x_{n}$. The GCD matrix $(S)_{f}$ of the set $S$ with respect to the function $f$ is the $n \times n$ matrix with $f\left(\operatorname{gcd}\left(x_{i}, x_{j}\right)\right)$ as its 
ij entry. This definition originates from the seminal paper [14 by H. J. S. Smith published in 1876. For more information about GCD and related matrices, see [1, 6, 10, 13.

Definition 3.1. An arithmetical function $f: \mathbb{Z}^{+} \rightarrow \mathbb{R}$ is positive definite if the GCD matrix $\left[f\left(\operatorname{gcd}\left(x_{i}, x_{j}\right)\right)\right]$ is positive semidefinite for all choices of points $\left\{x_{1}, x_{2}, \ldots, x_{n}\right\} \subset \mathbb{Z}^{+}$and all $n=1,2, \ldots$

Remark 3.1. Arithmetical function $f$ is positive definite if and only if the GCD matrix $(S)_{f}$ succeeds the corresponding zero matrix with respect to the Löwner order for all finite nonempty sets $S \subset \mathbb{Z}$.

Example 3.1. Let $\delta \in \mathcal{A}$ with $\delta(1)=1$ and $\delta(m)=0$ for all $m>1$ (the function $\delta$ is the identity element with respect to the Dirichlet convolution). Let $S=\{1,2\}$. Then

$$
(S)_{\delta}=\left[\begin{array}{ll}
\delta(\operatorname{gcd}(1,1)) & \delta(\operatorname{gcd}(1,2)) \\
\delta(\operatorname{gcd}(2,1)) & \delta(\operatorname{gcd}(2,2))
\end{array}\right]=\left[\begin{array}{ll}
\delta(1) & \delta(1) \\
\delta(1) & \delta(2)
\end{array}\right]=\left[\begin{array}{ll}
1 & 1 \\
1 & 0
\end{array}\right] .
$$

This matrix is not positive semidefinite, since $\operatorname{det}(S)_{\delta}=-1$, and thus $\delta$ is not a positive definite function.

Example 3.2. The Möbius function $\mu$ is defined as follows:

- $\mu(m)=(-1)^{k}$ if $p^{2} \nmid m$ for any prime number $p$ and $k$ is the number of the prime factors of $m$,

- $\mu(m)=0$ if $p^{2} \mid m$ for some prime number $p$.

Take any prime number $p$ and set $S=\{p\}$. We obtain $(S)_{\mu}=[\mu(p)]=[-1]$. Thus the function $\mu$ is not positive definite.

Example 3.3. Let $\alpha \in \mathbb{R}$. We define $N^{\alpha}(m)=m^{\alpha}$ for all $m \in \mathbb{Z}^{+}$.

1. Let $\alpha>0$. It is a well-known fact (see e.g. [4) that in this case the matrix $(S)_{N^{\alpha}}=\left[\operatorname{gcd}\left(x_{i}, x_{j}\right)^{\alpha}\right]$ is positive definite for all finite nonempty sets $S \subset \mathbb{Z}^{+}$. Thus $N^{\alpha}$ is a positive definite function for all $\alpha>0$.

2. Let $\alpha<0$ and $S=\left\{x_{1}, x_{2}\right\}$ with $x_{1} \mid x_{2}$. In this case

$$
(S)_{N^{\alpha}}=\left[\begin{array}{ll}
N^{\alpha}\left(x_{1}\right) & N^{\alpha}\left(x_{1}\right) \\
N^{\alpha}\left(x_{1}\right) & N^{\alpha}\left(x_{2}\right)
\end{array}\right]=\left[\begin{array}{ll}
x_{1}^{\alpha} & x_{1}^{\alpha} \\
x_{1}^{\alpha} & x_{2}^{\alpha}
\end{array}\right] .
$$

Now $\operatorname{det}(S)_{N^{\alpha}}=\left(x_{1} x_{2}\right)^{\alpha}-\left(x_{1}^{2}\right)^{\alpha}<0$. Thus $N^{\alpha}$ is not a positive definite function for any $\alpha<0$.

3. For $\alpha=0$ we denote $N^{0}=\zeta$ and have $\zeta(m)=1$ for all $m \in \mathbb{Z}^{+}$(the function $\zeta$ is the identity element with respect to the usual product). For any finite nonempty set $S \subset \mathbb{Z}$ the matrix $(S)_{\zeta}$ is an $n \times n$ matrix with all elements equal to 1 . It has two distinct eigenvalues: 0 with multiplicity $n-1$ and $n$ with multiplicity 1 . The matrix $(S)_{\zeta}$ is positive semidefinite and thus $\zeta$ is a positive definite function. 


\section{Positive definiteness properties for arithmeti- cal functions}

In this section we investigate various basic properties that follow directly from the definition of a positive definite arithmetical function. We continue to assume that $S$ is ordered as in the previous section: $x_{1}<x_{2}<\cdots<x_{n}$.

Theorem 4.1. Let $f \in \mathcal{A}$ be a positive definite function. Then

1. $f(m) \geq 0$ for all $m \in \mathbb{Z}^{+}$,

2. $k \mid m \Rightarrow f(k) \leq f(m)$ for all $k, m \in \mathbb{Z}^{+}$.

Proof. Let $m \in \mathbb{Z}^{+}$. The first claim follows by setting $S=\{m\}$, which yields the $1 \times 1$ GCD matrix $(S)_{f}=[f(m)]$. This matrix needs to be positive semidefinite, and therefore $f(m) \geq 0$.

Suppose next that $k \mid m$. In this case we choose $S=\{k, m\}$ to obtain the GCD matrix

$$
(S)_{f}=\left[\begin{array}{ll}
f(k) & f(k) \\
f(k) & f(m)
\end{array}\right] .
$$

The determinant of this matrix is equal to $f(k) f(m)-f(k)^{2}=f(k)(f(m)-$ $f(k)) \geq 0$. From this we deduce by distinguishing the cases in which $f(k)$ is 0 and $\neq 0$, that $f(m) \geq f(k)$.

Corollary 4.1. If $f \in \mathcal{A}$ is a positive definite function, then $f(m) \geq f(1) \geq 0$ for all $m \in \mathbb{Z}^{+}$.

Theorem 4.2. A function $f \in \mathcal{A}$ is positive definite if and only if the GCD matrix $\left(S_{m}\right)_{f}$ of the set $S_{m}=\{1,2, \ldots, m\}$ is positive semidefinite for all $m=$ $1,2, \ldots$

Proof. The implication $\Rightarrow$ is trivial, and thus it suffices to show the direction $\Leftarrow$. Suppose that the matrix $\left(S_{m}\right)_{f}$ of the set $S_{m}=\{1,2, \ldots, m\}$ is positive semidefinite for all $m=1,2, \ldots$ Let $S=\left\{x_{1}, x_{2}, \ldots, x_{n}\right\}$ be an arbitrary subset of $\mathbb{Z}^{+}$. Let $m$ be a positive integer with $x_{n} \leq m$. Now the GCD matrix $(S)_{f}$ of the set $S$ is a principal submatrix of the GCD matrix $\left(S_{m}\right)_{f}$ of the set $\{1,2, \ldots, m\}$. Since every principal submatrix of a positive semidefinite matrix is positive semidefinite, see [8, Observation 7.1.2], we may deduce that the matrix $(S)_{f}$ is positive semidefinite.

Theorem 4.3. A function $f \in \mathcal{A}$ is positive definite if and only if $(f * \mu)(k) \geq 0$ for all $k \in \mathbb{Z}^{+}$.

Proof. By Theorem 4.2, it suffices to show that the GCD matrix $\left(S_{m}\right)_{f}$ of the set $S_{m}=\{1,2, \ldots, m\}$ is positive semidefinite for all $m \in \mathbb{Z}^{+}$if and only if $(f * \mu)(k) \geq 0$ for all $k \in \mathbb{Z}^{+}$. Let $m \in \mathbb{Z}^{+}$. First we recall the well-known factorization

$$
\left(S_{m}\right)_{f}=E D E^{T}
$$


where $E$ is the $m \times m$ matrix with

$$
e_{i j}= \begin{cases}1 & \text { if } j \mid i \\ 0 & \text { otherwise }\end{cases}
$$

and $D=\operatorname{diag}((f * \mu)(1),(f * \mu)(2), \ldots,(f * \mu)(m))$. Since $E$ is a triangular matrix with all of its diagonal elements equal to 1, by Sylvester's Law of Inertia (see [8, Theorem 4.5.8]) we may deduce that the matrix $\left(S_{m}\right)_{f}$ is positive semidefinite if and only if the matrix $D$ is positive semidefinite. The claim follows from this.

Remark 4.1. Neither the argument used in the proof of Theorem 4.2 nor the idea of using $L D L^{T}$ factorization in determining the inertias of GCD type matrices are entirely new - both of them appear in the article [11] from the year 2004 by J. S. Ovall. The $L D L^{T}$ factorization itself originates from Rajarama Bhat [12] and Bourque and Ligh [4]. The factorization has also other applications, see e.g. [7.

Theorem 4.4. Let $f, g \in \mathcal{A}$ be positive definite functions. Then

1. af is a positive definite function for all $a \geq 0$,

2. $f+g$ is a positive definite function,

3. $f g$ is a positive definite function,

4. $f * g$ is a positive definite function.

Proof. It is clear that $(S)_{a f}=a(S)_{f}$ and $(S)_{f+g}=(S)_{f}+(S)_{g}$. Thus the properties 1 and 2 follow from the fact that every nonnegative linear combination of positive semidefinite matrices is positive semidefinite. Since $(S)_{f g}=(S)_{f}$ ○ $(S)_{g}$, the property 3 follows from the observation that the Hadamard product of two positive semidefinite matrices is positive semidefinite - see [8, Theorem 7.5.3]. We prove property 4 by showing that $((f * g) * \mu)(k) \geq 0$ for all $k \in \mathbb{Z}^{+}$. The associativity of the Dirichlet convolution yields

$$
((f * g) * \mu)(k)=(f *(g * \mu))(k)=\sum_{d \mid k} \underbrace{f(d)}_{\geq 0} \underbrace{(g * \mu)\left(\frac{k}{d}\right)}_{\geq 0} \geq 0 .
$$

Remark 4.2. It is easy to see that in the proof of Theorem 4.4 part 4 it suffices that one of the functions $f$ and $g$ is positive definite and the values of the other are nonnegative.

The following corollary is an immediate consequence of Theorem 4.4 
Corollary 4.2. Suppose that $f \in \mathcal{A}$ is positive definite. Then the functions

$$
f^{r}=\underbrace{f \cdot f \cdots f}_{r \text { times }} \quad \text { and } \quad f^{* r}=\underbrace{f * f * \cdots * f}_{r \text { times }}
$$

are positive definite for all $r=1,2,3, \ldots$.

It is also interesting to consider how positive definiteness of arithmetical functions behaves with respect to different inverse operations.

Theorem 4.5. Let $f \in \mathcal{A}$ be a positive definite function.

1. If $-f$ is also a positive definite function, then $f(m)=0$ for all $m \in \mathbb{Z}^{+}$.

2. If $f^{-1}=\frac{1}{f}$ exists and is also a positive definite function, then there exists $a \in \mathbb{R}$ such that $f(m)=a$ for all $m \in \mathbb{Z}^{+}$.

3. If $f^{*(-1)}$ (the Dirichlet inverse of $f$ ) exists, then it cannot be positive definite.

Proof. 1. The first part follows directly from the simple fact that if both $A$ and $-A$ are positive definite, then $A$ must be equal to the zero matrix. And if the GCD matrix of any finite nonempty set $S \subset \mathbb{Z}^{+}$with respect to the function $f$ is the zero matrix, then $f$ must be the constant function zero.

2. If the function $\frac{1}{f}$ exists and is positive definite, then we must have $f(m)>$ 0 for all $m \in \mathbb{Z}^{+}$. Let $m$ be an arbitrary integer greater than 1 and let $S=\{1, m\}$. Since $f$ and $\frac{1}{f}$ are positive definite, both of the GCD matrices

$$
(S)_{f}=\left[\begin{array}{ll}
f(1) & f(1) \\
f(1) & f(m)
\end{array}\right] \quad \text { and } \quad(S)_{\frac{1}{f}}=\left[\begin{array}{cc}
\frac{1}{f(1)} & \frac{1}{f(1)} \\
\frac{1}{f(1)} & \frac{1}{f(m)}
\end{array}\right]
$$

are positive semidefinite. The determinants of these matrices must be nonnegative, in other words,

$$
f(1)(f(m)-f(1)) \geq 0 \quad \text { and } \quad \frac{1}{f(1)}\left(\frac{1}{f(m)}-\frac{1}{f(1)}\right) \geq 0 .
$$

Since $f(1)>0$, the first inequality yields $f(m) \geq f(1)$ and the second implies that $f(1) \geq f(m)$. Thus we must have $f(m)=f(1)$ for any positive integer $m$.

3. If $f^{*(-1)}$ exists and $f$ is positive definite, then we have $f(1)>0$ and $f^{*(-1)}(1)=\frac{1}{f(1)}>0$. If $f(m)=0$ for all $m>1$, then there exists a positive real number $a$ such that $f=a \delta$, where $\delta$ is the arithmetical function defined in Example 3.1. Like the function $\delta$, the function $f$ is not positive definite. 
Assume next that $f(m)>0$ for some $m>1$. Let $m_{0}$ be the smallest positive integer such that $m_{0}>1$ and $f\left(m_{0}\right)>0$. We obtain

$$
\begin{aligned}
0 & =\delta\left(m_{0}\right)=\left(f * f^{*(-1)}\right)\left(m_{0}\right)=\sum_{d \mid m_{0}} f(d) f^{*(-1)}\left(\frac{m_{0}}{d}\right) \\
& =\underbrace{f(1)}_{>0} f^{*(-1)}\left(m_{0}\right)+\underbrace{f\left(m_{0}\right)}_{>0} \underbrace{f^{*(-1)}(1)}_{>0} .
\end{aligned}
$$

This means that we must have $f^{*(-1)}\left(m_{0}\right)<0$, and therefore $f^{*(-1)}$ cannot be positive definite.

\section{A partial order on the set of arithmetical func- tions}

Notation 5.1. Let $f$ and $g$ be arithmetical functions. If the function $g-f$ is positive definite we shall write $f \preceq g$.

Theorem 5.1. $f \preceq g$ if and only if the matrix $(S)_{g}-(S)_{f}$ is positive semidefinite for all finite nonempty sets $S \subset \mathbb{Z}^{+}$(in other words, $f \preceq g$ if and only if $(S)_{f} \leqslant(S)_{g}$ for all finite nonempty sets $S \subset \mathbb{Z}^{+}$, where $\leqslant$is the Löwner order).

Proof. By definition, $g-f$ is positive definite if and only if the matrix $(S)_{g-f}=$ $(S)_{g}-(S)_{f}$ is positive semidefinite for all sets $S=\left\{x_{1}, x_{2}, \ldots, x_{n}\right\} \subset \mathbb{Z}^{+}$and for all $n=1,2, \ldots$. Furthermore, this is equivalent to the statement that the matrix $(S)_{f}$ precedes the matrix $(S)_{g}$ in the sense of the Löwner order.

Theorem 5.2. The relation $\preceq$ is a partial order.

Proof. - For any $f \in \mathcal{A}$ the matrix $(S)_{f}-(S)_{f}=\mathbf{0}$ is positive semidefinite for all finite nonempty sets $S \subset \mathbb{Z}^{+}$. Thus $\preceq$ is reflexive.

- Suppose that $f \preceq g$ and $g \preceq f$. Thus for any finite nonempty set $S \subset \mathbb{Z}^{+}$ both of the matrices $(S)_{g}-(S)_{f}$ and $(S)_{f}-(S)_{g}$ are positive semidefinite, which implies that $(S)_{f}=(S)_{g}$. Therefore $f\left(x_{i}\right)=g\left(x_{i}\right)$ for all $x_{i} \in S$ and we must have $f=g$ (since $S$ is an arbitrary set).

- Suppose that $f \preceq g$ and $g \preceq h$. Let $S \subset \mathbb{Z}^{+}$. Now the matrices $(S)_{g}-(S)_{f}$ and $(S)_{h}-(S)_{g}$ are positive semidefinite and

$$
(S)_{h}-(S)_{f}=\left((S)_{h}-(S)_{g}\right)+\left((S)_{g}-(S)_{f}\right) .
$$

Thus $(S)_{h}-(S)_{f}$ is positive semidefinite and we must have $f \preceq h$.

The following results now follow directly from Theorems 4.1 and 4.3 .

Corollary 5.1. Suppose that $f \preceq g$. Then 
1. $f(m) \leq g(m)$ for all $m \in \mathbb{Z}^{+}$,

2. $k \mid m \Rightarrow g(k)-f(k) \leq g(m)-f(m)$ for all $k, m \in \mathbb{Z}^{+}$.

Corollary 5.2. Function $f \preceq g$ if and only if

$$
((g-f) * \mu)(k)=(g * \mu)(k)-(f * \mu)(k) \geq 0
$$

for all $k \in \mathbb{Z}$.

At this point it is natural to consider how our newly defined relation $\preceq$ relates to different function operations.

Theorem 5.3. Suppose that $0 \preceq f_{1} \preceq g_{1}$ and $0 \preceq f_{2} \preceq g_{2}$. Then

1. $0 \preceq f_{1} f_{2} \preceq g_{1} g_{2}$,

2. $0 \preceq f_{1} * f_{2} \preceq g_{1} * g_{2}$.

Proof. 1. We need to show that for any finite nonempty set $S \subset \mathbb{Z}^{+}$the matrix

$$
(S)_{f_{1} f_{2}}-(S)_{g_{1} g_{2}}=(S)_{f_{1}} \circ(S)_{f_{2}}-(S)_{g_{1}} \circ(S)_{g_{2}}
$$

is positive semidefinite. Since $\mathbf{0} \preceq(S)_{f_{1}} \preceq(S)_{g_{1}}$ and $\mathbf{0} \preceq(S)_{f_{2}} \preceq(S)_{g_{2}}$, the claim follows from [8, p. 475, Problem 4].

2. In the second case it is more convenient to use Corollary 5.2 and show that for all $k \in \mathbb{Z}^{+}$we have

$$
\left(\left(f_{1} * f_{2}\right) * \mu\right)(k) \leq\left(\left(g_{1} * g_{2}\right) * \mu\right)(k) .
$$

Let $k \in \mathbb{Z}^{+}$. By using the associativity of the Dirichlet convolution and Corollaries 5.1 and 5.2 we obtain

$$
\begin{aligned}
& \left(\left(f_{1} * f_{2}\right) * \mu\right)(k)=\left(f_{1} *\left(f_{2} * \mu\right)\right)(k)=\sum_{d \mid k} \overbrace{\leq g_{1}(d)}^{\overbrace{f_{1}(d)}} \overbrace{\underbrace{\left(f_{2} * \mu\right)\left(\frac{k}{d}\right)}_{\leq\left(g_{2} * \mu\right)\left(\frac{k}{d}\right)}}^{\geq 0} \\
& \leq \sum_{d \mid k} g_{1}(d)\left(g_{2} * \mu\right)\left(\frac{k}{d}\right)=\left(g_{1} *\left(g_{2} * \mu\right)\right)(k)=\left(\left(g_{1} * g_{2}\right) * \mu\right)(k) .
\end{aligned}
$$

Thus we have shown that $f_{1} * f_{2} \preceq g_{1} * g_{2}$. The property $0 \preceq f_{1} * f_{2}$ follows from Theorem 4.4.

Corollary 5.3. Suppose that $0 \preceq f \preceq g$. Then for all $r=1,2, \ldots$ we have

1. $0 \preceq f^{r} \preceq g^{r}$,

2. $0 \preceq f^{* r} \preceq g^{* r}$. 
Example 5.1. Recall that $\delta(1)=1$ and $\delta(m)=0$ for all $m>1$ and that $\zeta(m)=1$ for all $m \in \mathbb{Z}^{+}$. Since $\zeta(2)=1>0=\delta(2)$, clearly $\zeta \npreceq \delta$. Let us show that also $\delta \npreceq \zeta$. Consider the set $S=\{2,3,6\}$. We obtain

$$
(S)_{\zeta-\delta}=(S)_{\zeta}-(S)_{\delta}=\left[\begin{array}{lll}
1 & 1 & 1 \\
1 & 1 & 1 \\
1 & 1 & 1
\end{array}\right]-\left[\begin{array}{lll}
0 & 1 & 0 \\
1 & 0 & 0 \\
0 & 0 & 0
\end{array}\right]=\left[\begin{array}{lll}
1 & 0 & 1 \\
0 & 1 & 1 \\
1 & 1 & 1
\end{array}\right]
$$

The eigenvalues of this matrix are $1,1+\sqrt{2}$ and $1-\sqrt{2}<0$. Therefore the matrix $(S)_{\zeta-\delta}$ is not positive semidefinite and thus we cannot have $\delta \preceq \zeta$. It is also possible to consider the set $S=\{1,2,3,4,5,6\}$. In this case

$$
(S)_{\zeta-\delta}=\left[\begin{array}{cccccc}
0 & 0 & 0 & 0 & 0 & 0 \\
0 & 1 & 0 & 1 & 0 & 1 \\
0 & 0 & 1 & 0 & 0 & 1 \\
0 & 1 & 0 & 1 & 0 & 1 \\
0 & 0 & 0 & 0 & 1 & 0 \\
0 & 1 & 1 & 1 & 0 & 1
\end{array}\right] .
$$

The smallest eigenvalue of this matrix is approximately -0.4812 , and therefore the matrix is not positive semidefinite and we may deduce that $\delta \npreceq \zeta$.

Definition 5.1. Arithmetical function $f$ is said to be multiplicative if

$$
f(k m)=f(k) f(m)
$$

for all $k, m \in \mathbb{Z}^{+}$with $\operatorname{gcd}(k, m)=1$.

The values of a multiplicative function are completely determined by the values on prime powers. In fact, if $m=p_{1}^{a_{1}} p_{2}^{a_{2}} \cdots p_{k}^{a_{k}}$, then

$$
f(m)=f\left(p_{1}^{a_{1}} p_{2}^{a_{2}} \cdots p_{k}^{a_{k}}\right)=f\left(p_{1}^{a_{1}}\right) f\left(p_{2}^{a_{2}}\right) \cdots f\left(p_{k}^{a_{k}}\right) .
$$

The Möbius function $\mu$ is multiplicative, and the Dirichlet convolution of multiplicative functions is also multiplicative, see e.g. XXXX. Thus if $f \in \mathcal{A}$ is multiplicative and we wish to show that $f$ is positive definite, i.e. that $(f * \mu)(k) \geq 0$ for all $k \in \mathbb{Z}^{+}$, then it suffices to show that $(f * \mu)\left(p^{a}\right) \geq 0$ for any prime number $p$ and for all $a \in \mathbb{Z}^{+}$.

Example 5.2. The Jordan totient function $J_{\alpha}$ is defined as

$$
J_{\alpha}(m)=m^{\alpha} \prod_{p \mid m}\left(1-\frac{1}{p^{\alpha}}\right),
$$

where $m=p_{1}^{a_{1}} p_{2}^{a_{2}} \cdots p_{k}^{a_{k}}$. If $\alpha \geq 1$, then for any $a \geq 2$ we have

$$
\begin{aligned}
& \left(J_{\alpha} * \mu\right)\left(p^{a}\right)=\sum_{d \mid p^{a}} J_{\alpha}(d) \mu\left(\frac{p^{a}}{d}\right)=J_{\alpha}\left(p^{a}\right)-J_{\alpha}\left(p^{a-1}\right) \\
& =p^{\alpha a}-p^{\alpha(a-1)}-p^{\alpha(a-1)}+p^{\alpha(a-2)}=p^{\alpha(a-2)}\left(p^{2 \alpha}-2 p^{\alpha}+1\right) \\
& =p^{\alpha(a-2)}\left(p^{\alpha}-1\right)^{2} \geq 0
\end{aligned}
$$


and for $a=1$ we obtain

$$
\left(J_{\alpha} * \mu\right)(p)=J_{\alpha}(p)-1=p^{\alpha}-1-1=p^{\alpha}-2 \geq 0 .
$$

By multiplicativity this shows that $J_{\alpha}$ is positive definite for $\alpha \geq 1$. In particular, the Euler totient function $\phi=J_{1}$ is positive definite. Since $\left(J_{\alpha} * \mu\right)(2)=$ $2^{\alpha}-2<0$ for $\alpha<1$, we see that $J_{\alpha}$ is not positive definite for $\alpha<1$.

By utilizing multiplicativity in a similar manner it is possible to show that for $\alpha, \beta \geq 0$,

$$
J_{\alpha} \preceq J_{\beta} \Leftrightarrow\left(J_{\alpha} * \mu\right)(k) \leq\left(J_{\beta} * \mu\right)(k) \forall k \in \mathbb{Z}^{+} \Leftrightarrow \alpha \leq \beta .
$$

Example 5.3. In Example 3.3 it was shown that the power function $N^{\alpha}$ is positive definite for all $\alpha \geq 0$. With the aid of multiplicativity (as in Example 5.2 it is possible to show that for $\alpha, \beta \geq 0$,

$$
N^{\alpha} \preceq N^{\beta} \Leftrightarrow \alpha \leq \beta .
$$

Example 5.4. The divisor function $\sigma_{\alpha}$ is defined as $\sigma_{\alpha}(m):=\sum_{d \mid m} d^{\alpha}$, or alternatively $\sigma_{\alpha}=N^{\alpha} * \zeta$. The function $\sigma_{\alpha}$ is positive definite for all $\alpha \in \mathbb{R}$, and for any $\alpha, \beta \in \mathbb{R}$ we have

$$
\sigma_{\alpha} \preceq \sigma_{\beta} \Leftrightarrow \alpha \leq \beta .
$$

The positive definiteness of the function $\sigma_{\alpha}$ can easily be shown by using Theorem 4.3 , since

$$
\sigma_{\alpha} * \mu=\left(N^{\alpha} * \zeta\right) * \mu=N^{\alpha} *(\zeta * \mu)=N^{\alpha}
$$

and $N^{\alpha}(k) \geq 0$ for all $\alpha \in \mathbb{R}$ and $k \in \mathbb{Z}^{+}$. The other claim follows similarly, since

$$
\left(\sigma_{\beta}-\sigma_{\alpha}\right) * \mu=\left(\sigma_{\beta} * \mu\right)-\left(\sigma_{\alpha} * \mu\right)=N^{\beta}-N^{\alpha}
$$

and the values of this function are nonnegative if and only if $\alpha \leq \beta$.

Example 5.5. Let $\Omega(m)$ denote the total number of prime divisors of $m$ each counted according to its multiplicity (note that $\Omega(1)=0$ ). We prove the positive definiteness of the function $\Omega$ by showing that $(\Omega * \mu)(k) \geq 0$ for all $k \in \mathbb{Z}^{+}$.

If $k=1$, then $(\Omega * \mu)(k)=0$. Let $k=p_{1}^{k_{1}} p_{2}^{k_{2}} \cdots p_{r}^{k_{r}} \neq 1$ be the canonical factorization of $k$. Then

$$
\begin{gathered}
(\Omega * \mu)(k)=(\mu * \Omega)(k)=\sum_{d \mid k} \mu(d) \Omega\left(\frac{k}{d}\right) \\
=\left(\begin{array}{c}
\left.k_{1}+k_{2}+\cdots+k_{r}\right) \\
-\left(\left(k_{1}-1\right)+k_{2}+\cdots+k_{r}\right)-\left(k_{1}+\left(k_{2}-1\right)+\cdots+k_{r}\right)-\cdots \\
-\left(k_{1}+k_{2}+\cdots+\left(k_{r}-1\right)\right) \\
+\left(\left(k_{1}-1\right)+\left(k_{2}-1\right)+k_{3}+\cdots+k_{r}\right)+\cdots \\
\quad+\left(k_{1}+\cdots+k_{r-2}+\left(k_{r-1}-1\right)+\left(k_{r}-1\right)\right) \\
-\cdots
\end{array}\right.
\end{gathered}
$$


Denote $s=k_{1}+k_{2}+\cdots+k_{r}$. Then

$$
\begin{aligned}
(\Omega * \mu)(k) & =s-\left(\begin{array}{c}
r \\
1
\end{array}\right)(s-1)+\left(\begin{array}{l}
r \\
2
\end{array}\right)(s-2)+\cdots+(-1)^{r}\left(\begin{array}{l}
r \\
r
\end{array}\right)(s-r) \\
& =\sum_{i=0}^{r}(-1)^{i}\left(\begin{array}{l}
r \\
i
\end{array}\right)(s-i)=s \sum_{i=0}^{r}(-1)^{i}\left(\begin{array}{l}
r \\
i
\end{array}\right)-\sum_{i=0}^{r}(-1)^{i}\left(\begin{array}{l}
r \\
i
\end{array}\right) i .
\end{aligned}
$$

By the binomial theorem $\sum_{i=0}^{r}(-1)^{i}\left(\begin{array}{l}r \\ i\end{array}\right)=((-1)+1)^{r}$ and by formula (1.69) of Gould

$$
\sum_{i=0}^{r}(-1)^{i}\left(\begin{array}{c}
r \\
i
\end{array}\right) i=-r((-1)+1)^{r-1}
$$

(see, http://www.math.wvu.edu/ gould/Vol.2.PDF). Thus $(\Omega * \mu)(k)=1$ if $r=1$ (i.e., $k$ is a prime power $(\neq 1)$ ), and $(\Omega * \mu)(k)=0$ otherwise.

Example 5.6. Also for the generalized Liouville function $\lambda_{\alpha}(m)=\alpha^{\Omega(m)}$ it is possible to show that $\lambda_{\alpha}$ is positive definite if and only if $\alpha \geq 1$ and that for $\alpha, \beta \geq 1$,

$$
\alpha \leq \beta \Leftrightarrow \lambda_{\alpha} \preceq \lambda_{\beta} .
$$

In particular, the usual Liouville function $\lambda=\lambda_{-1}$ is not positive definite. These results can be proved by utilizing multiplicativity.

Example 5.7. For the generalized Dedekind function $\Psi_{\alpha}=N^{\alpha} * \mu^{2}$, where $\mu^{2}=\mu \cdot \mu=|\mu|$, it can be shown that $\Psi_{\alpha}$ is positive definite if and only if $\alpha \geq 0$ and that for $\alpha, \beta \geq 0$,

$$
\alpha \leq \beta \Leftrightarrow \Psi_{\alpha} \preceq \Psi_{\beta} .
$$

In particular, the usual Dedekind function $\Psi=\Psi_{1}$ is positive definite. Also these results can be shown by using multiplicativity.

Example 5.8. For $\alpha \geq 0$, we have

$$
J_{\alpha} \preceq N^{\alpha} \preceq \Psi_{\alpha} \preceq \sigma_{\alpha} .
$$

For $\alpha \geq 1$, we obtain

$$
\lambda_{\alpha} \preceq J_{\alpha} \preceq N^{\alpha} \preceq \Psi_{\alpha} \preceq \sigma_{\alpha} .
$$

These can be verified by applying the multiplicativity of the functions $\lambda_{\alpha} * \mu$, $J_{\alpha} * \mu, N^{\alpha} * \mu=J_{\alpha}, \Psi_{\alpha} * \mu$ and $\sigma_{\alpha} * \mu$. In particular (for $\alpha=1$ ),

$$
\phi\left(=J_{1}\right) \preceq N\left(=N^{1}\right) \preceq \Psi\left(=\Psi_{1}\right) \preceq \sigma\left(=\sigma_{1}\right) .
$$

\section{References}

[1] E. Altinisik, B. E. Sagan and N. Tuglu, GCD matrices, posets, and nonintersecting paths, Linear Multilinear Algebra 53 (2005) 75-84. 
[2] T. M. Apostol, Introduction to Analytic Number Theory (Springer-Verlag, 1976).

[3] R. Bhatia, Infinitely divisible matrices, Amer. Math. Monthly 113 No. 3 (2006) 221-235.

[4] K. Bourque and S. Ligh, Matrices associated with arithmetical functions, Linear and Multilinear Algebra 34 (1993) 261-267.

[5] Encyclopedic Dictionary of Mathematics, MIT Press, 1987.

[6] S. Hong and R. Loewy, Asymptotic behavior of eigenvalues of greatest common divisor matrices. Glasg. Math. J. 46 (2004) no. 3, 551-569.

[7] P. Ilmonen and P. Haukkanen, Smith meets Smith: Smith normal form of Smith matrix. Linear Multilinear Algebra 59 (2011) no. 5, 557-564.

[8] R. A. Horn and C. R. Johnson, Matrix Analysis, 1st ed. (Cambridge University Press, 1985).

[9] P. J. McCarthy, Introduction to Arithmetical Functions (Springer-Verlag, 1986).

[10] M. Mattila and P. Haukkanen, On the positive definiteness and eigenvalues of meet and join matrices, Discrete Math. 326 (2014) 9-19.

[11] J. S. Ovall, An analysis of GCD and LCM matrices via the $L D L^{T}$ factorization, Electron. J. Linear Algebra 11 (2004) 51-58.

[12] B. V. Rajarama Bhat, On greatest common divisor matrices and their applications, Linear Algebra Appl. 158 (1991) 77-97.

[13] J. Sándor and B. Crstici, Handbook of Number Theory II (Kluwer Academic, 2004).

[14] H. J. S. Smith, On the value of a certain arithmetical determinant, Proc. Lond. Math. Soc. 7 (1875-1876) 208-212. 